\title{
Codes and Invariant Theory
}

\author{
G. Nebe, \\ Abteilung Reine Mathematik, \\ Universität Ulm, \\ 89069 Ulm, Germany \\ Email: nebe@mathematik.uni-ulm.de \\ E. M. Rains, \\ Mathematics Department, \\ University of California Davis, \\ Davis, CA 95616, USA \\ Email: rains@math.ucdavis.edu \\ N. J. A. Sloane, \\ AT\&T Shannon Labs, \\ Florham Park, NJ 07932-0971, USA \\ Email: njas@research.att.com \\ January 1, 2003; revised October 6, 2003
}

\begin{abstract}
The main theorem in this paper is a far-reaching generalization of Gleason's theorem on the weight enumerators of codes which applies to arbitrary-genus weight enumerators of self-dual codes defined over a large class of finite rings and modules. The proof of the theorem uses a categorical approach, and will be the subject of a forthcoming book. However, the theorem can be stated and applied without using category theory, and we illustrate it here by applying it to generalized doubly-even codes over fields of characteristic 2 , doubly-even codes over $\mathbb{Z} / 2^{f} \mathbb{Z}$, and self-dual codes over the noncommutative ring $\mathbb{F}_{q}+\mathbb{F}_{q} u$, where $u^{2}=0$..
\end{abstract}

AMS 2000 Classification: Primary 94B05, 13A50; Secondary: $94 \mathrm{~B} 60$

\section{Introduction}

One of the most remarkable theorems in coding theory is Gleason's 1971 theorem [7] that the weight enumerator of a binary doubly-even self-dual code is an element of the polynomial ring generated by the weight enumerators of the Hamming code of length 8 and the Golay code of length 24. In the past thirty years many generalizations of this 
theorem have been given that apply to other familes of codes (cf. [13, [16]). Usually each new type of code is treated on an individual basis. We have recently found a far-reaching generalization of Gleason's result that applies simultaneously to arbitrary-genus weight enumerators of self-dual codes over a very large class of finite rings and modules.

The main result (Theorem 3.5 below) can be summarized as follows. Given a quasichain ring $R$ and a notion of self-duality for codes over a left $R$-module $V$, we construct a "Clifford-Weil" group $G$ such that the vector invariants of $G$ are spanned by the full weight enumerators of self-dual isotropic codes, and the polynomial invariants of $G$ are spanned by the complete weight enumerators of these codes.

In the case of genus- $m$ weight enumerators (for $m \geq 1$ ) of Type I binary self-dual codes, $G$ is the real Clifford group $\mathcal{C}_{m}$ of our earlier paper [13. If $C$ is a Type II binary self-dual code, $G$ is the complex Clifford group $\mathcal{X}_{m}$ of [13]. The case $m=1$ gives the original Gleason theorem (except for the specific identification of codes that generate the ring). For self-dual codes over $\mathbb{F}_{p}$ containing the all-ones vector (where $p$ is an odd prime) $G$ is the group $\mathcal{C}_{m}^{(p)}$ of [13, Section 7].

The proof of the main theorem will be the subject of our forthcoming book [14. The proof is best carried out via a categorical approach, using the concept of a form ring, a generalization of the corresponding concept from unitary $K$-theory [8]. However, it is not necessary to understand this theory to state and apply the theorem, and so it seems worthwhile publishing a short version that states the main result and gives some applications. To illustrate the theorem we will construct the Clifford-Weil groups for generalized doubly-even codes over fields of characteristic 2 (Section 5), doubly-even codes over $\mathbb{Z} / 2^{f} \mathbb{Z}$ (Section 6) , and self-dual codes over the noncommutative ring $\mathbb{F}_{q}+\mathbb{F}_{q} u$ where $u^{2}=0$ (Section [7). In Section 2 we define self-dual code, isotropic code and the Type of a code. Section 3 defines certain weight enumerators and gives the main theorem, while Section 4 studies the Clifford-Weil groups and their structure. For further details the reader is referred to 14 .

\section{Self-dual isotropic codes.}

Throughout the paper, $R$ will denote a ring (with unit element 1 ) and $V$ a left $R$-module.

Definition 2.1. A (linear) code $C$ of length $N$ over $V$ is an $R$-submodule of the left $R$-module $V^{N}$. In the classical language of coding theory, $V$ is the alphabet over which the code is defined, and $R$ is the ground ring (so if $c \in C$ and $r \in R, r c \in C$ ).

Coding theory usually deals with codes over a finite alphabet $V$. Therefore we will assume in the following that $V$ is a finite left $R$-module over the finite ring $R$. Some parts of the theory carry over to infinite rings, and so can be applied to self-dual lattices, for example (see [14]). But our explicit construction of the Clifford-Weil group only applies to the finite case. 
To express self-duality, we need a nonsingular bilinear form $\beta$ on $V$. Since $V$ is finite, $\beta$ can be chosen to have values in $\mathbb{Q} / \mathbb{Z}$. A bilinear form

$$
\beta \in \operatorname{Bil}(V, \mathbb{Q} / \mathbb{Z}):=\{\beta: V \times V \rightarrow \mathbb{Q} / \mathbb{Z} \mid \beta \text { is } \mathbb{Z} \text {-bilinear }\}
$$

is nonsingular if $v \mapsto \beta(v, \cdot)$ is an isomorphism of the abelian groups $V$ and $V^{*}=$ $\operatorname{Hom}(V, \mathbb{Q} / \mathbb{Z})$. The following definition is then the natural generalization of the usual notion of dual code (cf. [11]).

Definition 2.2. Let $C \leq V^{N}$ be a code and $\beta \in \operatorname{Bil}(V, \mathbb{Q} / \mathbb{Z})$ a nonsingular bilinear form. The dual code (with respect to $\beta$ ) is

$$
C^{\perp}:=\left\{x=\left(x_{1}, \ldots, x_{N}\right) \in V^{N} \mid \sum_{i=1}^{N} \beta\left(x_{i}, c_{i}\right)=0 \text { for all } c=\left(c_{1}, \ldots, c_{N}\right) \in C\right\} .
$$

$C$ is called self-dual (with respect to $\beta$ ) if $C=C^{\perp}$.

To express certain additional constraints on the code (that weights are divisible by 4 for binary codes, or that the code contains the all-ones vector $\mathbf{1}=(1, \ldots, 1)$, etc.) we use quadratic mappings, which we define to be sums of quadratic forms and linear forms on $V$, with values in $\mathbb{Q} / \mathbb{Z}$ :

Definition 2.3. Let $\operatorname{Quad}_{0}(V, \mathbb{Q} / \mathbb{Z}):=\{\phi: V \rightarrow \mathbb{Q} / \mathbb{Z} \mid \phi(0)=0$ and

$$
\phi(x+y+z)-\phi(x+y)-\phi(x+z)-\phi(y+z)+\phi(x)+\phi(y)+\phi(z)=0\} .
$$

Let $\Phi \subset \operatorname{Quad}_{0}(V, \mathbb{Q} / \mathbb{Z})$ and let $C \leq V^{N}$ be a code. Then $C$ is called isotropic (with respect to $\Phi)$ if $\phi(c):=\sum_{i=1}^{N} \phi\left(c_{i}\right)=0$ for all $c \in C$ and $\phi \in \Phi$.

Remarks. Let $V$ be a left $R$-module. Then

(a) $\operatorname{Bil}(V, \mathbb{Q} / \mathbb{Z})$ is a right $(R \otimes R)$-module via $\beta(r \otimes s)(v, w)=\beta(r v, s w)$ for all $\beta \in \operatorname{Bil}(V, \mathbb{Q} / \mathbb{Z}), v, w \in V, r, s \in R$.

(b) $\operatorname{Bil}(V, \mathbb{Q} / \mathbb{Z})$ has a natural involution $\tau$ defined by $\left(\beta^{\tau}\right)(v, w):=\beta(w, v)$ for all $\beta \in \operatorname{Bil}(V, \mathbb{Q} / \mathbb{Z}), v, w \in V$.

(c) For $r \in R$ define $[r] \in \operatorname{End}\left(\operatorname{Quad}_{0}(V, \mathbb{Q} / \mathbb{Z})\right)$ by $(\phi[r])(v)=\phi(r v)$ for $\phi \in$ $\operatorname{Quad}_{0}(V, \mathbb{Q} / \mathbb{Z}), v \in V$. This "action" satisfies $[r s]=[r][s]$ and

$$
[r+s+t]-[r+s]-[r+t]-[s+t]+[r]+[s]+[t]=0
$$

for all $r, s, t \in R$. We describe this situation by saying that we have made the group $\operatorname{Quad}_{0}(V, \mathbb{Q} / \mathbb{Z})$ into an $R$-qmodule.

(d) The mapping $[-1]$ is an involution on $\operatorname{Quad}_{0}(V, \mathbb{Q} / \mathbb{Z})$.

(e) There is a mapping $\mathbb{R}\}: \operatorname{Bil}(V, \mathbb{Q} / \mathbb{Z}) \rightarrow \operatorname{Quad}_{0}(V, \mathbb{Q} / \mathbb{Z})$ defined by $\{\beta\}(v):=$ $\beta(v, v)$ for all $\beta \in \operatorname{Bil}(V, \mathbb{Q} / \mathbb{Z}), v \in V$. 
(f) There is a mapping $\lambda: \operatorname{Quad}_{0}(V, \mathbb{Q} / \mathbb{Z}) \rightarrow \operatorname{Bil}(V, \mathbb{Q} / \mathbb{Z})$ defined by $(\lambda(\phi))(v, w):=$ $\phi(v+w)-\phi(v)-\phi(w)$ for all $\phi \in \operatorname{Quad}_{0}(V, \mathbb{Q} / \mathbb{Z}), v, w \in V$.

(g) Both mappings $\lambda$ and $\mathbb{\}}$ are $R$-qmodule homomorphisms, where $\operatorname{Bil}(V, \mathbb{Q} / \mathbb{Z})$ is regarded as an $R$-qmodule via $\beta[r](v, w):=\beta(r v, r w)$ for all $\beta \in \operatorname{Bil}(V, \mathbb{Q} / \mathbb{Z}), v, w \in V$, $r \in R$.

(h) For all $\beta \in \operatorname{Bil}(V, \mathbb{Q} / \mathbb{Z})$ and $\phi \in \operatorname{Quad}_{0}(V, \mathbb{Q} / \mathbb{Z})$ we have

$$
\begin{aligned}
\left\{\beta^{\tau}\right\} & =\{\beta\}, \\
\lambda(\phi)^{\tau} & =\lambda(\phi), \\
\lambda(\{\beta\}) & =\beta+\beta^{\tau}, \\
\phi[r+s]-\phi[r]-\phi[s] & =\{\lambda(\phi)(r \otimes s)\} .
\end{aligned}
$$

(i) For all $\beta \in \operatorname{Bil}(V, \mathbb{Q} / \mathbb{Z})$ and $\phi \in \operatorname{Quad}_{0}(V, \mathbb{Q} / \mathbb{Z})$ we have

$$
\lambda(\{\lambda(\phi)\})=2 \lambda(\phi) \text { and }\{\lambda(\{\beta \beta)\}=2\{\{\beta\} .
$$

We call $\beta \in \operatorname{Bil}(V, \mathbb{Q} / \mathbb{Z})$ admissible if $\beta$ is nonsingular and the $(1 \otimes R)$-submodule of $\operatorname{Bil}(V, \mathbb{Q} / \mathbb{Z})$ given by

$M:=\beta(1 \otimes R)=\left\{\beta_{r} \mid r \in R\right\}$, where $\beta(1 \otimes r)=\beta_{r}$ is defined by $\beta_{r}(v, w)=\beta(v, r w)$, is closed under $\tau$ and isomorphic to $R$, i.e. if

$$
\psi: r \mapsto \beta_{r}
$$

defines an isomorphism of right $R$-modules $\psi: R_{R} \rightarrow M_{1 \otimes R}$.

Note that any admissible $\beta$ defines an anti-automorphism $J$ of $R$ by $r \mapsto r^{J}$, where

$$
\beta(r v, w)=\beta\left(v, r^{J} w\right) \text { for all } v, w \in V .
$$

Let $\epsilon \in R$ be defined by $\beta(v, w)=\beta(w, \epsilon v)$ for all $v, w \in V$. Then $\epsilon^{J} r^{\left(J^{2}\right)} \epsilon=r$ for all $r \in R$. In particular $\epsilon^{J} \epsilon=1$ and since $R$ is finite, $\epsilon$ is a unit.

Definition 2.4. The quadruple $\rho:=(R, V, \beta, \Phi)$ is called a (finite representation of a) form ring if $R$ is a finite ring, $V$ is a finite left $R$-module, $\beta \in \operatorname{Bil}(V, \mathbb{Q} / \mathbb{Z})$ is an admissible bilinear form, and $\Phi \leq \operatorname{Quad}_{0}(V, \mathbb{Q} / \mathbb{Z})$ is a sub-R-qmodule such that $\{M\} \leq \Phi$ and $\lambda(\Phi) \leq M$, where $M:=\beta(1 \otimes R)$. Then if $C \leq V^{N}$ for some integer $N \geq 1$ is self-dual with respect to $\beta$ and isotropic with respect to $\Phi, C$ is called a (self-dual isotropic) code of Type $\rho$.

Remarks. (a) The definition of Type given in 14] uses a more abstract definition of a form ring. For our purposes here it is enough to work with the above concrete realization.

(b) The definition of Type given above (and the still more general version given in [14]), are far-reaching generalizations of the notion of Type used in [11, [16].

(c) The usual notion of form ring [8] corresponds directly to the case when $\lambda$ is injective; it is also closely connected to the dual case when \{\} is surjective. 


\section{Weight enumerators and Clifford-Weil groups.}

There are many kinds of weight enumerators of codes (see e.g. [16]). We introduce here only the complete weight enumerator, although analogues of our main theorem hold for full weight enumerators and other appropriate symmetrizations of the full weight enumerator.

Definition 3.1. Let $C \leq V^{N}$ be a code. The complete weight enumerator of $C$ is $\operatorname{cwe}(C):=\sum_{c \in C} \prod_{i=1}^{N} x_{c_{i}} \in \mathbb{C}\left[x_{v} \mid v \in V\right]$.

The genus-m complete weight enumerator of $C$ is

$$
\operatorname{cwe}_{m}(C):=\sum_{\left(c^{(1)}, \ldots, c^{(m)}\right) \in C^{m}} \prod_{i=1}^{N} x_{\left(c_{i}^{(1)}, \ldots, c_{i}^{(m)}\right)} \in \mathbb{C}\left[x_{v} \mid v \in V^{m}\right] .
$$

Remark 3.2. The genus- $m$ complete weight enumerator of $C$ can be obtained from the complete weight enumerator of $C(m):=C \otimes R^{m} \leq V^{N} \otimes R^{m} \cong V^{m N}$. For if we identify $V^{m N}$ with $\left(V^{m}\right)^{N}$ and consider $C(m)$ as a code in $\left(V^{m}\right)^{N}$, then cwe $_{m}(C)=\operatorname{cwe}(C(m))$.

Note that $C(m)$ is a code for which the ground ring is $\operatorname{Mat}_{m}(R)$, the ring of $m \times m$ matrices with entries in $R$. This is one of the main reasons why we allow noncommutative ground rings: even when we consider genus- $m$ weight enumerators for classical binary codes, $\operatorname{Mat}_{m}\left(\mathbb{F}_{2}\right)$ arises naturally as the ground ring.

Let $\rho=(R, V, \beta, \Phi)$ be a form ring. Let $C \leq V^{N}$ be a self-dual isotropic code of Type $\rho$. Then the complete weight enumerator cwe $(C)$ is invariant under the substitutions

$$
\begin{gathered}
\rho(r): x_{v} \mapsto x_{r v}, \text { for all } r \in R^{*} \quad \begin{array}{c}
\text { (since } C \text { is a code), } \\
\rho(\phi): x_{v} \mapsto \exp (2 \pi i \phi(v)) x_{v}, \text { for all } \phi \in \Phi
\end{array} \quad \text { (since } C \text { is isotropic), }
\end{gathered}
$$

as well as the MacWilliams transformation (cf. [11, [16]):

$$
h: x_{v} \mapsto \sqrt{|V|}^{-1} \sum_{w \in V} \exp (2 \pi i \beta(w, v)) x_{w} \quad\left(\text { since } C=C^{\perp}\right) .
$$

Subsidiary transformations can be derived from $h$. If $R=\mathbb{Z} / 6 \mathbb{Z}$, for example, we also obtain the MacWilliams transformations modulo 2 and modulo 3. For general rings $R$, one can similarly construct further MacWilliams transformations using symmetric idempotents, as we now demonstrate.

Example 3.3. Let $V^{\prime}=\iota R$ for some idempotent $\iota \in R$. Then $V^{\prime}$ admits a nonsingular $J$-Hermitian form if and only if there is an isomorphism of right $R$-modules

$$
\kappa: \iota R \cong \iota^{J} R,
$$

in which case we say that the idempotent is symmetric. Note that any such isomorphism $\kappa$ has the form

$$
\kappa(\iota x)=v_{\iota} x, \kappa^{-1}\left(\iota^{J} x\right)=u_{\iota} x,
$$

where $u_{\iota} \in \iota R \iota^{J}$ and $v_{\iota} \in \iota^{J} R \iota$ satisfy $u_{\iota} v_{\iota}=\iota, v_{\iota} u_{\iota}=\iota^{J}$. 
If $\iota=u_{\iota} v_{\iota}$ is a symmetric idempotent in $R$, then

$$
h_{\iota, v_{\iota}}: x_{v} \mapsto \sqrt{|\iota V|}^{-1} \sum_{w \in \iota V} \exp \left(2 \pi i \beta\left(w, v_{\iota} v\right)\right) x_{w+(1-\iota) v}
$$

is the partial MacWilliams transformation corresponding to $\iota V$.

Definition 3.4. Let $\rho=(R, V, \beta, \Phi)$ be a form ring. Then the associated Clifford-Weil group is

$$
\left.\mathcal{C}(\rho):=\left\langle\rho(r), \rho(\phi), h_{\iota, v_{\iota}}\right| r \in R^{*}, \phi \in \Phi, \iota \text { symmetric idempotent in } R\right\rangle,
$$

a subgroup of $\mathrm{GL}_{|V|}(\mathbb{C})$.

The above discussion shows that the complete weight enumerator of a self-dual isotropic code of Type $\rho$ is invariant under the action of the group $\mathcal{C}(\rho)$. If $G$ is any subgroup of $\mathrm{GL}_{n}(\mathbb{C})$ we let

$$
\operatorname{Inv}(G):=\left\{p \in \mathbb{C}\left[x_{1}, \ldots, x_{n}\right] \mid p(g X)=p(X) \text { for all } g \in G\right\}
$$

denote the invariant ring of $G$.

Now we can state our main theorem, part (i) of which is clear from the above considerations and the MacWilliams identities. We cannot at present prove part (ii) for arbitrary finite rings, but need to make some additional assumptions, for example that $R$ is a chain ring (i.e. the left ideals in $R$ are linearly ordered by inclusion) or, more generally, a quasi-chain ring, by which we mean a direct product of matrix rings over chain rings.

Theorem 3.5. Let $\rho=(R, V, \beta, \Phi)$ be a form ring.

(i) If $C \leq V^{N}$ is a self-dual isotropic code then cwe $(C) \in \operatorname{Inv}(\mathcal{C}(\rho))$.

(ii) If $R$ is a finite quasi-chain ring then $\operatorname{Inv}(\mathcal{C}(\rho))$ is spanned by complete weight enumerators of self-dual isotropic codes of Type $\rho$ :

$$
\left.\operatorname{Inv}(\mathcal{C}(\rho))=\langle\operatorname{cwe}(C)| C \text { self-dual, isotropic code in } V^{N}, N \geq 1\right\rangle .
$$

To deal with higher-genus weight enumerators we introduce the associated CliffordWeil group $\mathcal{C}_{m}(\rho):=\mathcal{C}\left(\rho \otimes R^{m}\right)$ of genus $m$. By Remark 3.2 the invariant ring of $\mathcal{C}_{m}(\rho)$ is spanned by the genus- $m$ weight enumerators of self-dual codes of Type $\rho$.

Let $\rho:=(R, V, \beta, \Phi)$ be a form ring. By Morita theory (see [14]), this corresponds to a unique form ring

$$
\operatorname{Mat}_{m}(\rho)=\rho \otimes R^{m}:=\left(\operatorname{Mat}_{m}(R), V \otimes R^{m}, \beta^{(m)}, \Phi_{m}\right)
$$


which we call a matrix ring for the form ring $\rho$. Here $\beta^{(m)}$ is the bilinear form on $V^{m}=V \otimes R^{m}$ (admissible for $\left.\operatorname{Mat}_{m}(R)\right)$ defined by

$$
\beta^{(m)}\left(\left(v_{1}, \ldots, v_{m}\right),\left(w_{1}, \ldots, w_{m}\right)\right)=\sum_{i=1}^{m} \beta\left(v_{i}, w_{i}\right) .
$$

$\beta^{(m)}$ generates the $\operatorname{Mat}_{m}(R)$-module $M_{m}:=\beta^{(m)}\left(1 \otimes \operatorname{Mat}_{m}(R)\right)$, and

$$
\Phi_{m}=\left\{\left(\begin{array}{cccc}
\phi_{1} & m_{12} & \ldots & m_{1 m} \\
& \ddots & \ddots & \vdots \\
& & \ddots & m_{m-1, m} \\
& & & \phi_{m}
\end{array}\right) \mid \phi_{1}, \ldots, \phi_{m} \in \Phi, m_{i j} \in M\right\}
$$

is a set of upper triangular matrices, where

$$
\left(\begin{array}{cccc}
\phi_{1} & m_{12} & \cdots & m_{1 m} \\
& \ddots & \ddots & \vdots \\
& & \ddots & m_{m-1, m} \\
& & & \phi_{m}
\end{array}\right)\left(v_{1}, \ldots, v_{m}\right)=\sum_{i=1}^{m} \phi_{i}\left(v_{i}\right)+\sum_{i<j} m_{i j}\left(v_{i}, v_{j}\right)
$$

One easily sees that $\Phi_{m}$ is a $\operatorname{sub-Mat}_{m}(R)$-qmodule of $\operatorname{Quad}_{0}\left(V \otimes R^{m}\right)$. The involution $J_{m}$ on $\operatorname{Mat}_{m}(R)$ acts as componentwise application of $J$ followed by transposition. The unit $\epsilon_{m} \in \operatorname{Mat}_{m}(R)$ is the scalar matrix $\epsilon I_{m}$.

\section{The structure of the Clifford-Weil groups.}

The group $\mathcal{C}(\rho)$ is a projective representation of a so-called hyperbolic co-unitary group $\mathcal{U}(R, \Phi)$, which we will define in terms of $R$, the involution $J$ and the $R$-qmodule $\Phi$. $\mathcal{U}(R, \Phi)$ is an extension of the linear $R$-module $\operatorname{ker}(\lambda) \oplus \operatorname{ker}(\lambda)$ by a certain group $G$ of $2 \times 2$ matrices over the ring $R$. This is one of two possible extensions of the unitary $K$-theoretic notion of "hyperbolic unitary group" [8] that applies to our generalized definition of form ring.

Let $\rho:=(R, V, \beta, \Phi)$ be a form ring and let $M:=\beta(1 \otimes R)$. Then, using the above construction of the $2 \times 2$-matrix ring for a form ring, $\Phi_{2}$ is a $\operatorname{Mat}_{2}(R)$ sub-qmodule of $\operatorname{Quad}_{0}\left(V \otimes R^{2}\right)$. In particular, $\Phi_{2}$ is a module for the unit group $\mathrm{GL}_{2}(R)$ of $\operatorname{Mat}_{2}(R)$, and we can form the semi-direct product $\mathrm{GL}_{2}(R) \ltimes \Phi_{2}$ of which $\mathcal{U}(R, \Phi)$ will be a subgroup. Applying $\lambda$ and $\psi$ componentwise, we get mappings $\lambda_{2}: \Phi_{2} \rightarrow \operatorname{Mat}_{2}(M)$ defined by $\lambda_{2}\left(\left(\begin{array}{cc}\phi_{1} & m \\ & \phi_{2}\end{array}\right)\right):=\left(\begin{array}{cc}\lambda\left(\phi_{1}\right) & m \\ m^{\tau} & \lambda\left(\phi_{2}\right)\end{array}\right)$ and $\psi_{2}: \operatorname{Mat}_{2}(R) \rightarrow \operatorname{Mat}_{2}(M)$.

Then $\mathcal{U}(R, \Phi)=$

$\left\{\left(\left(\begin{array}{ll}a & b \\ c & d\end{array}\right),\left(\begin{array}{cc}\phi_{1} & m \\ & \phi_{2}\end{array}\right)\right) \in \mathrm{GL}_{2}(R) \ltimes \Phi_{2} \mid \psi_{2}\left(\left(\begin{array}{cc}c^{J} a & c^{J} b \\ d^{J} a-1 & d^{J} b\end{array}\right)\right)=\lambda_{2}\left(\left(\begin{array}{cc}\phi_{1} & m \\ m^{\tau} & \phi_{2}\end{array}\right)\right)\right\}$. 
Remark. To describe the isomorphism type of $\mathcal{U}(R, \Phi)$, note that the projection

$$
\pi: \mathcal{U}(R, \Phi) \rightarrow \mathrm{GL}_{2}(R),(u, \phi) \mapsto u
$$

defines a group homomorphism. The kernel of $\pi$ is the set of all $(1, \phi) \in \mathcal{U}(R, \Phi)$, i.e.

$$
\operatorname{ker}(\pi)=\left\{\left(1,\left(\begin{array}{cc}
\phi_{1} & 0 \\
& \phi_{2}
\end{array}\right)\right) \mid \phi_{1}, \phi_{2} \in \Phi, \lambda\left(\phi_{1}\right)=\lambda\left(\phi_{2}\right)=0\right\}
$$

which is naturally isomorphic to $\operatorname{ker}(\lambda) \times \operatorname{ker}(\lambda)$. The image of $\pi$ is

$$
\left\{\left(\begin{array}{ll}
a & b \\
c & d
\end{array}\right) \in \operatorname{Mat}_{2}(R) \mid\left(\begin{array}{ll}
a^{J} & c^{J} \\
b^{J} & d^{J}
\end{array}\right)\left(\begin{array}{ll}
0 & 0 \\
1 & 0
\end{array}\right)\left(\begin{array}{ll}
a & b \\
c & d
\end{array}\right)-\left(\begin{array}{ll}
0 & 0 \\
1 & 0
\end{array}\right) \in \psi_{2}^{-1}\left(\lambda_{2}\left(\Phi_{2}\right)\right)\right\}
$$

In many important examples it is easy to describe the image of $\psi_{2}^{-1} \circ \lambda_{2}$ (for example, this may consist of all symmetric, skew-symmetric or hermitian elements) and so leads to an isomorphism of $\mathcal{U}(R, \Phi) / \operatorname{ker}(\pi)$ with (a subgroup of) a classical group.

Example 4.1. Let $R$ be one of the finite simple rings with involution shown in the following table, where $\operatorname{Mat}_{n}\left(\mathbb{F}_{q}\right)$ denotes the ring of $n \times n$ matrices over the field $\mathbb{F}_{q}$, with transposition denoted by $\mathrm{t}$. Then for an appropriate center $Z$,

$$
\mathcal{C}(\rho)=Z \cdot \mathcal{U}(R, \Phi)=Z \cdot(\operatorname{ker}(\lambda) \oplus \operatorname{ker}(\lambda)) \cdot \mathcal{G}(R, \Phi)
$$

where $\mathcal{G}(R, \Phi)$ is the classical group shown in the last column:

\begin{tabular}{|c|c|c|c|}
\hline$R$ & $J$ & $\epsilon$ & $\mathcal{G}(R, \Phi)$ \\
\hline $\operatorname{Mat}_{n}\left(\mathbb{F}_{q}\right) \oplus \operatorname{Mat}_{n}\left(\mathbb{F}_{q}\right)$ & $(r, s)^{J}=\left(s^{\mathrm{t}}, r^{\mathrm{t}}\right)$ & 1 & $\mathrm{GL}_{2 n}\left(\mathbb{F}_{q}\right)$ \\
\hline $\operatorname{Mat}_{n}\left(\mathbb{F}_{q^{2}}\right)$ & $r^{J}=\left(r^{q}\right)^{\mathrm{t}}$ & 1 & $U_{2 n}\left(\mathbb{F}_{q^{2}}\right)$ \\
\hline $\operatorname{Mat}_{n}\left(\mathbb{F}_{q}\right), q=p^{m}, p>2$ & $r^{J}=r^{\mathrm{t}}$ & 1 & $\operatorname{Sp}_{2 n}\left(\mathbb{F}_{q}\right)$ \\
\hline $\operatorname{Mat}_{n}\left(\mathbb{F}_{q}\right), q=p^{m}, p>2$ & $r^{J}=r^{\mathrm{t}}$ & -1 & $O_{2 n}^{+}\left(\mathbb{F}_{q}\right)$ \\
\hline $\operatorname{Mat}_{n}\left(\mathbb{F}_{q}\right), q=p^{m}, p=2$ & $\psi^{-1}(\lambda(\Phi))=\left\{r \in R \mid r^{J}=r\right\}$ & $\operatorname{Sp}_{2 n}\left(\mathbb{F}_{q}\right)$ \\
\hline $\operatorname{Mat}_{n}\left(\mathbb{F}_{q}\right), q=p^{m}, p=2$ & $\psi^{-1}(\lambda(\Phi))=\{0\}$ & \\
\hline
\end{tabular}

Recall that a ring $R$ is semiperfect (cf. [10, page 346]) if $R / \operatorname{rad} R$ is semisimple and idempotents of $R / \operatorname{rad} R$ lift to idempotents of $R$; in particular, all finite rings are semiperfect. One can show the following: 
Theorem 4.2. (See [14].) If $R$ is semiperfect, then the hyperbolic co-unitary group $\mathcal{U}(R, \Phi)$ is generated by the following elements:

$$
d(u, \phi):=\left(\left(\begin{array}{cc}
u^{-J} & u^{-J} \psi^{-1}(\lambda(\phi)) \\
0 & u
\end{array}\right),\left(\begin{array}{ll}
0 & 0 \\
& \phi
\end{array}\right)\right)
$$

for all $u \in R^{*}, \phi \in \Phi$, and

$$
H_{\iota, u_{\iota}, v_{\iota}}:=\left(\left(\begin{array}{cc}
1-\iota^{J} & v_{\iota} \\
-\epsilon^{-1} u_{\iota}^{J} & 1-\iota
\end{array}\right),\left(\begin{array}{cc}
0 & \psi(-\epsilon \iota) \\
0
\end{array}\right)\right)
$$

for all symmetric idempotents $\iota \in R$.

Then the projective representation $\mathcal{U}(R, \Phi) \rightarrow \mathcal{C}(\rho)$ is defined by

$$
d(u, \phi) \mapsto \rho(u) \rho(\phi)
$$

and

$$
H_{\iota, u_{\iota}, v_{\iota}} \mapsto h_{\iota, v_{\iota}} .
$$

Hence $\mathcal{C}(\rho)$ is an extension

$$
1 \rightarrow Z \rightarrow \mathcal{C}(\rho) \rightarrow \mathcal{U}(R, \Phi) \rightarrow 1
$$

where $Z$ consists of scalar matrices (since the projective representation can be seen to be irreducible). If Theorem 3.5 holds for $\rho$, then by invariant theory $Z \cong Z_{l}$ is cyclic of order $l$ where $l=\operatorname{gcd}\left\{N \mid\right.$ there exists $C \leq V^{N}$ of Type $\left.\rho\right\}$. In fact, for arbitrary $\rho$ (not necessarily satisfying Theorem 3.5), one can show that

$$
\begin{aligned}
|Z| & =\operatorname{gcd}\left\{N \geq 1 \mid \text { there exists } C \leq V^{N} \text { of Type } \rho\right\} \\
& =\min \left\{N \geq 1 \mid \text { there exists } C \leq V^{N} \text { of Type } \rho\right\} .
\end{aligned}
$$

\section{Doubly-even euclidean self-dual codes over $\mathbb{F}_{2^{f}}$.}

This and the next two sections will illustrate the above theory. For further details about this first section see [12. In [15] Quebbemann defines the notion of an even code over the field $k:=\mathbb{F}_{2^{f}}$ as follows. A code $C \leq k^{N}$ is called even if

$$
\sum_{i=1}^{N} c_{i}=0 \text { and } \sum_{i<j} c_{i} c_{j}=0, \quad \text { for all } c \in C .
$$

It is easy to see that even codes are self-orthogonal with respect to the usual bilinear form $\sum_{i=1}^{N} c_{i} c_{i}^{\prime}$. Moreover, identifying $k$ with $\mathbb{F}_{2}^{f}$ using a self-complementary (or traceorthonormal) basis, even codes remain even over $\mathbb{F}_{2}$. A self-dual even code in this sense 
is called a generalized doubly-even self-dual code. If $f=1$, even codes are precisely the classical doubly-even (or Type II) binary codes. If $f=2$, they are the Type II codes over $\mathbb{F}_{4}$ considered in [6].

The Type of these codes can be specified in the language of form rings as follows. Let $R=\mathbb{F}_{2^{f}}, V=R$ and $\beta: V \times V \rightarrow \frac{1}{2} \mathbb{Z} / \mathbb{Z}$ be defined by $\beta(x, y):=\frac{1}{2} \operatorname{tr}(x y)$, where $\operatorname{tr}$ denotes the trace from $\mathbb{F}_{2^{f}}$ to $\mathbb{F}_{2} \cong \mathbb{Z} / 2 \mathbb{Z}$. Then $\beta$ is admissible and $M:=\beta(1 \otimes R)=$ $\left\{\beta_{a}:=\beta(1 \otimes a) \mid a \in R\right\}$.

The quadratic forms will take values modulo 4 . Let $O:=\mathbb{Z}_{2}\left[\zeta_{2^{f}-1}\right]$ be the ring of integers in the unramified extension of degree $f$ of the 2 -adic numbers. Then $R \cong O / 2 O$. If $x \in R, x^{2}$ is uniquely determined modulo 4 , so squares of elements of $R$ can be considered as elements of $O / 4 O$. The usual trace $\operatorname{Tr}: O \rightarrow \mathbb{Z}$ maps $4 O$ into $4 \mathbb{Z}$. For $a \in R$ we define

$$
\phi_{a}: V \rightarrow \frac{1}{4} \mathbb{Z} / \mathbb{Z}, \phi_{a}(x):=\frac{1}{4} \operatorname{Tr}\left(a^{2} x^{2}\right) \in \operatorname{Quad}_{0}(V, \mathbb{Q} / \mathbb{Z}),
$$

and let $\Phi:=\left\{\phi_{a} \mid a \in R\right\}$. Then $(R, V, \beta, \Phi)$ is a form ring.

Theorem 5.1. Codes of Type $\rho$ are exactly the generalized doubly-even self-dual codes in the sense of (5.1).

Proof. Let $C \leq \mathbb{F}_{2^{f}}^{N}$ be an even code in the sense of (5.1). Since $\lambda$ is surjective, it is enough to show that $\sum_{i=1}^{N} \phi_{a}\left(c_{i}\right)=0$ for all $c \in C$. Now $\sum_{i=1}^{N} c_{i}=0$, therefore as an element of $O / 4 O$ the square

$$
\left(\sum_{i=1}^{N} c_{i}\right)^{2}=\sum_{i=1}^{N} c_{i}^{2}+2 \sum_{i<j} c_{i} c_{j}=0 \in O / 4 O .
$$

Since $\sum_{i<j} c_{i} c_{j}=0$ it follows that $\sum_{i=1}^{N} c_{i}^{2}=0 \in O / 4 O$.

To obtain the other inclusion, let $C$ be a code of Type $\rho$. By the nondegeneracy of the trace form, $\sum_{i=1}^{N} c_{i}^{2}=0$ in $O / 4 O$. Therefore by (5.2),$\left(\sum_{i=1}^{N} c_{i}\right)^{2} \equiv 0(\bmod 2 O)$ and hence also $\sum_{i=1}^{N} c_{i}=0 \in \mathbb{F}_{2^{f}}$. Then $\left(\sum_{i=1}^{N} c_{i}\right)^{2} \equiv 0(\bmod 4 O)$ and (5.2) implies that $\sum_{i<j} c_{i} c_{j}=0$.

We next compute the Clifford-Weil groups of arbitrary genus for these codes.

Theorem 5.2. Let $\mathcal{C}_{m}(\rho)$ be the Clifford-Weil group of genus $m$ corresponding to the form ring $\rho$ above. Then

$$
\mathcal{C}(\rho) \cong Z \cdot\left(k^{m} \oplus k^{m}\right) \cdot \mathrm{Sp}_{2 m}(k) \cong Z \cdot 2^{2 m f} \cdot \mathrm{Sp}_{2 m}\left(2^{f}\right),
$$

where $Z \cong Z_{4}$ if $f:=\left[k: \mathbb{F}_{2}\right]$ is even, $Z \cong Z_{8}$ if $f:=\left[k: \mathbb{F}_{2}\right]$ is odd. 
Proof. From Section $4, \mathcal{C}_{m}(\rho)$ has an epimorphic image

$$
\mathcal{U}\left(\operatorname{Mat}_{m}(R), \Phi_{m}\right) \cong\left(k^{m} \oplus k^{m}\right) \cdot \operatorname{Sp}_{2 m}(k)
$$

The kernel $Z$ of this epimorphism is a cyclic group consisting of scalar matrices. Since the invariant ring of $\mathcal{C}_{m}(\rho)$ is spanned by weight enumerators of self-dual isotropic codes $C$, the order of $Z$ is the greatest common divisor of the lengths of these self-dual isotropic codes. Since the codes are self-dual, they all contain the all-ones vector $\mathbf{1}$. This vector spans an isotropic $k$-space if and only if the length $N$ of the code is divisible by 4 . Therefore $|Z|$ is divisible by 4 .

First consider the case when $f$ is even. The code $Q_{4}$ with generator matrix

$$
\left[\begin{array}{lllc}
1 & 1 & 1 & 1 \\
0 & 1 & \omega & \omega^{2}
\end{array}\right]
$$

where $\omega \in \mathbb{F}_{4} \backslash \mathbb{F}_{2}$, is an isotropic self-dual code of length 4 over $\mathbb{F}_{4}$. Extending scalars, one gets an isotropic self-dual code $k \otimes_{\mathbb{F}_{4}} Q_{4}$ of length 4 for all $k$ which are of even degree over $\mathbb{F}_{2}$. Hence in this case $Z \cong Z_{4}$.

If $f$ is odd, then any self-dual isotropic code $C \leq k^{N}$ yields a doubly-even self-dual binary code $\tilde{C} \leq \mathbb{F}_{2}^{f N}$. The length $f N$ of $\tilde{C}$ is necessarily divisible by 8 . Since $f$ is odd, this implies that $N$ is divisible by 8 and hence in this case $Z \cong Z_{8}$.

The case $k=\mathbb{F}_{2}$. We obtain the classical doubly-even binary codes; the highergenus Clifford-Weil groups are the complex Clifford groups of [13].

The case $k=\mathbb{F}_{4}$. Let $k=\mathbb{F}_{4}=\left\{0,1, \omega, \omega^{2}\right\}$. Then

$$
G:=\mathcal{C}(\rho) \cong\left(Z_{4} \mathrm{Y}_{D_{8}} \mathrm{Y}_{D_{8}}\right) \cdot \mathrm{Alt}_{5}
$$

(where $\mathrm{Y}$ denotes a central product) is generated by

$$
\rho(\omega)=\left(\begin{array}{llll}
1 & 0 & 0 & 0 \\
0 & 0 & 0 & 1 \\
0 & 1 & 0 & 0 \\
0 & 0 & 1 & 0
\end{array}\right), h=\frac{1}{2}\left(\begin{array}{rrrr}
1 & 1 & 1 & 1 \\
1 & 1 & -1 & -1 \\
1 & -1 & -1 & 1 \\
1 & -1 & 1 & -1
\end{array}\right)
$$

and $\rho(\phi)=\operatorname{diag}(1,-1, i, i) . G$ is a subgroup of index 2 in the complex reflection group $G_{29}$ (No. 29 in [17]). The Molien series of $G$ (cf. [11], [16]) is

$$
\frac{1+t^{40}}{\left(1-t^{4}\right)\left(1-t^{8}\right)\left(1-t^{12}\right)\left(1-t^{20}\right)} .
$$

Primary invariants of $G$ (which generate the invariant ring of $G_{29}$ ) can be taken to be the complete weight enumerators of the extended quadratic residue codes $Q_{N}$ of lengths $N=4,8,12,20$ over $\mathbb{F}_{4}$. The elements in $G_{29} \backslash G$ act as the Frobenius automorphism 
$\omega \mapsto \bar{\omega}$ on the weight enumerators of these codes. A weight enumerator $p_{C}$ is invariant under $G_{29}$ if $p_{C}=\overline{p_{C}}$. To get the full invariant ring of $G$, a further code is needed, a self-dual even code $C_{40}$ of length 40 over $\mathbb{F}_{4}$, for which the complete weight enumerator is not invariant under the Frobenius automorphism. Such a code is constructed in [2].

The case $k=\mathbb{F}_{8}$. In this case we will just give the Molien series of $\mathcal{C}(\rho)$. This can be written as $f(t) / g(t)$, where

$$
\begin{aligned}
f(t):=1 & +5 t^{16}+77 t^{24}+300 t^{32}+908 t^{40}+2139 t^{48}+3808 t^{56}+5864 t^{64} \\
& +8257 t^{72}+10456 t^{80}+12504 t^{88}+14294 t^{96}+15115 t^{104} \\
& +15115 t^{112}+14294 t^{120}+12504 t^{128}+10456 t^{136}+8257 t^{144} \\
& +5864 t^{152}+3808 t^{160}+2139 t^{168}+908 t^{176}+300 t^{184} \\
& +77 t^{192}+5 t^{200}+t^{216}
\end{aligned}
$$

and

$$
g(t):=\left(1-t^{8}\right)^{2}\left(1-t^{16}\right)^{2}\left(1-t^{24}\right)^{2}\left(1-t^{56}\right)\left(1-t^{72}\right) .
$$

It would clearly be hopeless to attempt to find codes whose weight enumerators generate this ring. This phenomenon is typical (compare 9]): it is the exception rather than the rule for these rings of invariants to have a simple description in terms of codes.

\section{Doubly-even self-dual codes over $\mathbb{Z} / 2^{f} \mathbb{Z}$.}

Let $R:=\mathbb{Z} / 2^{f} \mathbb{Z}$ and let $C \leq R^{N}$ be a code of length $N$. Then the dual code $C^{\perp}:=\{x \in$ $R^{N} \mid \sum_{i=1}^{N} x_{i} c_{i}=0$, for all $\left.c \in C\right\}$. $C$ is called doubly-even if $\sum_{i=1}^{N} c_{i}^{2} \equiv 0\left(\bmod 2^{f+1}\right)$. To describe the class of doubly-even self-dual codes over $R$ in the language of form rings let $V:=R$, and define $\beta: V \times V \rightarrow \frac{1}{2^{f}} \mathbb{Z} / \mathbb{Z}$ by $\beta(x, y):=\frac{1}{2^{f}} x y$ and $\phi_{0}: V \rightarrow \frac{1}{2^{f+1}} \mathbb{Z} / \mathbb{Z}$ by $\phi_{0}(x):=\frac{1}{2^{f+1}} x^{2}$. Let $\Phi$ be the $R$-qmodule generated by $\phi_{0}$ and define the form ring

$$
\rho_{a}:=(R, V, \beta, \Phi) .
$$

To express the additional property that $C$ contains the all-ones vector, let $\varphi: V \rightarrow$ $\frac{1}{2^{f}} \mathbb{Z} / \mathbb{Z}$ be defined by $\varphi(x):=\frac{1}{2^{f}} x$ and let $\Phi_{0}$ be the $R$-qmodule spanned by $\phi_{0}$ and $\varphi$. Then define the form ring

$$
\rho_{b}:=\left(R, V, \beta, \Phi_{0}\right) .
$$

Note that $\operatorname{ker}\left(\lambda_{a}\right)=\left\langle 2^{f} \phi_{0}\right\rangle \cong \mathbb{Z} / 2 \mathbb{Z}$, whereas $\operatorname{ker}\left(\lambda_{b}\right)=\langle\varphi\rangle \cong R$, since $2^{f-1} \varphi=2^{f} \phi_{0}$. Since the involution on $R$ is trivial and $\epsilon=1$, we find that

$$
\pi\left(\mathcal{U}_{m}(R, \Phi)\right)=\left\{A \in \operatorname{Mat}_{2 m}(R) \mid A^{\operatorname{tr}}\left(\begin{array}{cc}
0 & -I_{m} \\
I_{m} & 0
\end{array}\right) A=\left(\begin{array}{cc}
0 & -I_{m} \\
I_{m} & 0
\end{array}\right)\right\} \cong \operatorname{Sp}_{2 m}(R) .
$$


Theorem 6.1. For $x \in\{a, b\}$, let $l_{x}=\operatorname{gcd}\left\{N \mid\right.$ there exists a code $C \leq V^{N}$ of Type $\left.\rho_{x}\right\}$. The Clifford-Weil groups of genus $m$ are extensions

$$
\mathcal{C}\left(\rho_{a}\right) \cong Z_{l_{a}} \cdot\left(Z_{2}^{m} \times Z_{2}^{m}\right) \cdot \operatorname{Sp}_{2 m}(R)
$$

and

$$
\mathcal{C}\left(\rho_{b}\right) \cong Z_{l_{b}} \cdot\left(R^{m} \times R^{m}\right) \cdot \operatorname{Sp}_{2 m}(R)
$$

As we show in [14], $l_{a}=8$ and $l_{b}=\max \left\{2^{f+1}, 8\right\}$.

The case $f=2$. We identify $R$ with $\mathbb{Z} / 4 \mathbb{Z}=\{0,1,2,3\}$ and let $\zeta:=\exp (2 \pi i / 8)$ be a primitive eighth root of unity in $\mathbb{C}$, with $i=\zeta^{2}$. Then

$$
\mathcal{C}\left(\rho_{a}\right)=\left\langle\left(\begin{array}{llll}
1 & 0 & 0 & 0 \\
0 & 0 & 0 & 1 \\
0 & 0 & 1 & 0 \\
0 & 1 & 0 & 0
\end{array}\right), \frac{1}{2}\left(\begin{array}{rrrr}
1 & 1 & 1 & 1 \\
1 & i & -1 & -i \\
1 & -1 & 1 & -1 \\
1 & -i & -1 & i
\end{array}\right), \operatorname{diag}(1, \zeta,-1, \zeta)\right\rangle
$$

of order $\left|\mathcal{C}\left(\rho_{a}\right)\right|=1536$, and

$$
\mathcal{C}\left(\rho_{b}\right)=\left\langle\mathcal{C}\left(\rho_{a}\right), \operatorname{diag}(1, i,-1,-i)\right\rangle
$$

of order $\left|\mathcal{C}\left(\rho_{b}\right)\right|=6144$. Furthermore, $\mathcal{C}\left(\rho_{a}\right) \cong Z_{8} \times\left(Z_{2} \times Z_{2}\right) . S L_{2}(\mathbb{Z} / 4 \mathbb{Z})$ and $\mathcal{C}\left(\rho_{b}\right) \cong$ $Z_{8} \times\left(Z_{4} \times Z_{4}\right) \cdot S L_{2}(\mathbb{Z} / 4 \mathbb{Z})$. The Molien series are

$$
\operatorname{Molien}\left(\mathcal{C}\left(\rho_{a}\right)\right)=\frac{\left(1+t^{8}\right)\left(1+t^{16}\right)^{2}}{\left(1-t^{8}\right)^{3}\left(1-t^{24}\right)}
$$

and

$$
\operatorname{Molien}\left(\mathcal{C}\left(\rho_{b}\right)\right)=\frac{\left(1+t^{16}\right)\left(1+t^{32}\right)}{\left(1-t^{8}\right)^{2}\left(1-t^{16}\right)\left(1-t^{24}\right)}
$$

(see [16]). We will interpret these rings in terms of complete weight enumerators of self-dual isotropic codes. If $p$ is a prime power $p \equiv \pm 1(\bmod 8)$, let $\tilde{Q R}(p)$ denote the extended quadratic residue code of length $p+1$ over $\mathbb{Z} / 4 \mathbb{Z}$ (see for example [3]). With the correct definition of extension, $\tilde{Q R}(p)$ contains the all-ones vector. Let $d_{8}, c_{16}, d_{16}$ be the codes of Type $\rho_{b}$ (see [4]) with generator matrices

$$
d_{8}=\left[\begin{array}{l}
13100102 \\
13010210 \\
13001021 \\
22000000 \\
20222000
\end{array}\right], \quad c_{16}=\left[\begin{array}{l}
111111111111111 \\
1011111100001000 \\
1101001111000100 \\
1110101010100010 \\
0000111111100001 \\
0000020000022002 \\
0000002000022222 \\
0000000200002202 \\
0000000020000222 \\
0000000002020202 \\
0000000000220022
\end{array}\right], \quad d_{16}=\left[\begin{array}{l}
111111111111111 \\
1110000023000000 \\
1101000002300000 \\
1100100022230000 \\
1100010002223000 \\
1100001022222300 \\
1100000102222230 \\
1011111102222221
\end{array}\right] .
$$


Let $p_{1}:=\operatorname{cwe}(\tilde{Q R}(7)), p_{2}:=\operatorname{cwe}\left(d_{8}\right), p_{3}:=\operatorname{cwe}\left(c_{16}\right), p_{4}:=\operatorname{cwe}\left(d_{16}\right), p_{5}:=\operatorname{cwe}(\tilde{Q R}(23))$. Then

$$
\operatorname{Inv}\left(\mathcal{C}\left(\rho_{b}\right)\right)=\mathbb{C}\left[p_{1}, p_{2}, p_{3}, p_{5}\right]\left(1+p_{4}\right)\left(1+p_{6}\right),
$$

where $p_{6}$ is the weight enumerator of a certain code $e_{32}$ of Type $\rho_{b}$ and length 32 . We have an explicit description of a code $e_{16}$ that works, but it has no structure and we do not give it here. It would be nice to have a better example.

To find additional generators for the invariant ring of $\mathcal{C}\left(\rho_{a}\right)$, let $e_{8}^{\prime}$ and $d_{8}^{\prime}$ be the codes of Type $\rho_{a}$ obtained from $\tilde{Q R}(7)$ resp. $d_{8}$ by multiplying one column by $3 \in \mathbb{Z} / 4 \mathbb{Z}$, and let $p_{1 a}:=\operatorname{cwe}\left(e_{8}^{\prime}\right)$ and $p_{2 a}:=\operatorname{cwe}\left(d_{8}^{\prime}\right)$ be their complete weight enumerators. We also need three further codes of Type $\rho_{a}$ : two of length 24 and one of length 32, with complete weight enumerators $f_{1}, f_{2}, f_{3}$ respectively. We have examples of such codes, but again they have no structure and we do not give them here. With this notation we find that

$$
\operatorname{Inv}\left(\mathcal{C}\left(\rho_{b}\right)\right)=\mathbb{C}\left[p_{1}, p_{2}, p_{1 a}, p_{5}\right]\left(1+p_{2 a}+p_{3}+p_{4}+f_{1}+f_{2}+f_{3}+p_{2 a} f_{3}\right)
$$

\section{$7 \quad$ Self-dual codes over $\mathbb{F}_{q^{2}}+\mathbb{F}_{q^{2}} u$}

In this section we study self-dual codes over the $\operatorname{ring} R=\mathbb{F}_{q^{2}}+\mathbb{F}_{q^{2}} u$, for $q=p^{f}$, where $u^{2}=0$ and $u a=a^{q} u$ for all $a \in \mathbb{F}_{q^{2}}$. These are certainly "non-classical" codes.

We have $R \cong \mathcal{M} / p \mathcal{M}$, where $\mathcal{M}$ is the maximal order in the quaternion division algebra over the unramified extension of degree $f$ of the $p$-adic numbers. The most important case is $q=2$. In this special case, self-dual codes have been studied by Bachoc [1] in connection with the construction of interesting modular lattices, and Gaborit [5] has found a mass formula.

To construct self-dual codes, we define an $R$-valued Hermitian form $R^{N} \times R^{N} \rightarrow R$ by $(x, y):=\sum_{i=1}^{N} x_{i} \overline{y_{i}}$, where $-: R \rightarrow R$ is the involution defined by $\overline{a+b u}:=a^{q}-b u$. Then

$$
\left(a^{\prime}+b^{\prime} u\right) \overline{(a+b u)}=a^{\prime} a^{q}+\left(a b^{\prime}-b a^{\prime}\right) u,
$$

for all $a, b, a^{\prime}, b^{\prime} \in \mathbb{F}_{q^{2}}$. A code $C \leq R^{N}$ is self-dual if $C=C^{\perp}:=\left\{v \in R^{N} \mid(v, c)=\right.$ 0 for all $c \in C\}$.

To express this self-duality in our language of Types, we need a form ring. Let $\beta: R \times R \rightarrow \frac{1}{p} \mathbb{Z} / \mathbb{Z}$ be the bilinear form defined by

$$
\beta\left(a^{\prime}+b^{\prime} u, a+b u\right):=\frac{1}{p} \operatorname{Tr}\left(a b^{\prime}-a^{\prime} b\right),
$$

where $\operatorname{Tr}$ denotes the trace from $\mathbb{F}_{q^{2}}$ to $\mathbb{F}_{p} \cong \mathbb{Z} / p \mathbb{Z}$. Let $M:=\beta(1 \otimes R)$, where the right action of $R \otimes R$ on $M$ is left multiplication on the arguments:

$$
m\left((r+s u) \otimes\left(r^{\prime}+s^{\prime} u\right)\right)\left(a+b u, a^{\prime}+b^{\prime} u\right)=m\left((r+s u)(a+b u),\left(r^{\prime}+s^{\prime} u\right)\left(a^{\prime}+b^{\prime} u\right)\right),
$$


for $m \in M$ and where $r+s u, r^{\prime}+s^{\prime} u, a+b u, a^{\prime}+b^{\prime} u$ are elements of $R$. Let $\psi: R_{R} \rightarrow M_{1 \otimes R}$ be the $R$-module isomorphism defined by $\psi(1):=\beta$. The involution $J$ induced by $\beta$ is given by $(r+s u)^{J}=r-s^{q} u$, and $\epsilon=-1$ (since $\beta$ is skew-symmetric).

Define the $\frac{1}{p} \mathbb{Z} / \mathbb{Z}$-valued quadratic form $\phi_{0}: R \rightarrow \frac{1}{p} \mathbb{Z} / \mathbb{Z}$ by

$$
\phi_{0}(a+b u):=\frac{1}{p} \operatorname{Tr}_{\mathbb{F}_{q} / \mathbb{F}_{p}}\left(a a^{q}\right)
$$

and let $\Phi:=\left\{\phi_{0}[r] \mid r \in R\right\}$. The mapping $\mathbb{R}: M \rightarrow \Phi$ is the obvious diagonal evaluation

$$
\{\beta(1 \otimes(r+s u))\}(a+b u):=\beta(1 \otimes(r+s u))(a+b u, a+b u)=-\operatorname{Tr}\left(s a a^{q}\right) .
$$

Since $\mathbb{R}$ is surjective, this defines a unique mapping $\lambda: \Phi \rightarrow M$, satisfying

$$
\lambda\{m\}=m+\tau(m),
$$

for all $m \in M$. To find $\lambda\left(\phi_{0}\right)$, we choose $\omega \in \mathbb{F}_{q^{2}} \backslash \mathbb{F}_{q}$ with $\omega+\omega^{q}=-1$. Then $\phi_{0}=\{\beta(1 \otimes \omega u)\}$,

$$
\lambda\left(\phi_{0}\right)=m_{0}(1 \otimes \omega u)+\tau(\beta(1 \otimes \omega u))=\beta(1 \otimes u)
$$

and therefore $\psi^{-1}\left(\lambda\left(\phi_{0}\right)\right)=u$. Identifying $\mathbb{F}_{p}$ with $\frac{1}{p} \mathbb{Z} / \mathbb{Z}$, we obtain $\mathbb{Q} / \mathbb{Z}$-valued quadratic and bilinear forms. This defines a form ring $\rho=(R, V, \beta, \Phi)$, and the self-dual codes $C \leq R^{N}$ defined above are precisely the codes of Type $\rho$.

The hyperbolic co-unitary group $\mathcal{U}(R, \Phi)$ contains a normal subgroup $N$ for which the quotient is a subgroup of $\mathcal{U}\left(\mathbb{F}_{q^{2}},\{0\}\right)$. In fact, since $R=\mathbb{F}_{q^{2}}+\mathbb{F}_{q^{2}} u, \mathcal{U}(R, \Phi)$ has a subgroup $H \cong O_{2}^{+}\left(\mathbb{F}_{q^{2}}\right)$ consisting of the elements

$$
\left\{\left(\left(\begin{array}{ll}
a & b \\
c & d
\end{array}\right),\left(\begin{array}{cc}
0 & \psi(c b) \\
\psi(d a-1) & 0
\end{array}\right)\right) \mid a, b, c, d \in \mathbb{F}_{q^{2}}, c a=d b=0, c b+d a=1\right\} .
$$

$H$ is isomorphic to $\mathcal{U}\left(\mathbb{F}_{q^{2}},\{0\}\right)$ and is a complement to the normal subgroup $\mathcal{U}((u), \Phi)$ given by

$$
\left\{\left(\left(\begin{array}{cc}
1+a u & b u \\
c u & 1+d u
\end{array}\right), \operatorname{diag}\left(\phi_{0}\left[c^{\prime}\right], \phi_{0}\left[b^{\prime}\right]\right)\right) \mid c, b \in \mathbb{F}_{q}, a, d \in \mathbb{F}_{q^{2}}, a=d^{q}\right\},
$$

which is isomorphic to $\mathbb{F}_{q^{2}} \oplus \mathbb{F}_{q} \oplus \mathbb{F}_{q}$. Therefore

$$
\mathcal{U}(R, \Phi) \cong\left(\mathbb{F}_{q^{2}} \oplus \mathbb{F}_{q} \oplus \mathbb{F}_{q}\right) \rtimes O_{2}^{+}\left(\mathbb{F}_{q^{2}}\right)
$$

Example 7.1. Let $q:=2, R=\mathbb{F}_{4}+\mathbb{F}_{4} u$. Then the hyperbolic co-unitary group $\mathcal{U}(R, \Phi)$ is generated by

$$
g_{1}:=\left(\left(\begin{array}{cc}
\omega & 0 \\
0 & \omega^{2}
\end{array}\right), 0\right), g_{2}:=\left(\left(\begin{array}{cc}
1+u & 0 \\
0 & 1+u
\end{array}\right), 0\right)
$$




$$
g_{3}:=\left(\left(\begin{array}{ll}
1 & u \\
0 & 1
\end{array}\right),\left(\begin{array}{cc}
0 & 0 \\
& \phi_{0}
\end{array}\right)\right), h:=\left(\left(\begin{array}{ll}
0 & 1 \\
1 & 0
\end{array}\right),\left(\begin{array}{ll}
0 & 1 \\
& 0
\end{array}\right)\right) .
$$

Since $\lambda$ is injective, $\mathcal{U}(R, \Phi)$ is isomorphic to its image under the projection of the first component. This image contains a normal subgroup $N \cong \mathbb{F}_{4}+\mathbb{F}_{2}+\mathbb{F}_{2}$ generated by

$$
\left(\begin{array}{cc}
1+\omega u & 0 \\
0 & 1+\omega^{2} u
\end{array}\right),\left(\begin{array}{cc}
1+u & 0 \\
0 & 1+u
\end{array}\right),\left(\begin{array}{ll}
1 & u \\
0 & 1
\end{array}\right),\left(\begin{array}{ll}
1 & 0 \\
u & 1
\end{array}\right) .
$$

The quotient group is isomorphic to $S_{3}$, generated by the matrices

$$
\left(\begin{array}{cc}
\omega & 0 \\
0 & \omega^{2}
\end{array}\right), \quad\left(\begin{array}{ll}
0 & 1 \\
1 & 0
\end{array}\right)
$$

Hence

$$
\mathcal{U}(R, \Phi) \cong\left(\left(C_{2}\right)^{2} \times\left(C_{2}\right)^{2}\right) \rtimes S_{3}
$$

where $S_{3}$ acts faithfully on one copy $\left(C_{2}\right)^{2}$ and with kernel $C_{3}$ on the other copy.

The Molien series of $\mathcal{C}(\rho)$ is

$$
\frac{f(t)}{\left(1-t^{2}\right)^{5}\left(1-t^{3}\right)\left(1-t^{4}\right)^{6}\left(1-t^{6}\right)^{4}},
$$

where

$$
\begin{aligned}
f(t):=1 & +t+4 t^{2}+3 t^{3}+53 t^{4}+104 t^{5}+458 t^{6}+858 t^{7}+2474 t^{8}+4839 t^{9} \\
& +10667 t^{10}+19018 t^{11}+34193 t^{12}+55481 t^{13}+86078 t^{14} \\
& +125990 t^{15}+173466 t^{16}+230402 t^{17}+287430 t^{18}+346462 t^{19} \\
& +393648 t^{20}+431930 t^{21}+450648 t^{22}+450648 t^{23}+431930 t^{24} \\
& +393648 t^{25}+346462 t^{26}+287430 t^{27}+230402 t^{28}+173466 t^{29} \\
& +125990 t^{30}+86078 t^{31}+55481 t^{32}+34193 t^{33}+19018 t^{34} \\
& +10667 t^{35}+4839 t^{36}+2474 t^{37}+858 t^{38}+458 t^{39}+104 t^{40} \\
& +53 t^{41}+3 t^{42}+4 t^{43}+t^{44}+t^{45}
\end{aligned}
$$

Various interesting symmetrizations are possible:

a) Symmetrizing by the action of $\mathbb{F}_{4}^{*}=\langle\omega\rangle$ yields a matrix group $\mathcal{C}_{(7)}(\rho) \cong\left(C_{2}\right)^{2} \rtimes S_{3}$ (a non-faithful $S_{3}$-action) of degree 7 , with Molien series

$$
\frac{1+t^{2}+9 t^{4}+21 t^{6}+41 t^{8}+43 t^{10}+43 t^{12}+23 t^{14}+10 t^{16}}{(1-t)\left(1-t^{2}\right)\left(1-t^{4}\right)^{3}\left(1-t^{6}\right)^{2}} .
$$

b) The unit group $R^{*}$ has three orbits on $R$ namely $\{0\}, R^{*}, u R^{*}$. Symmetrizing by $R^{*}$ gives a matrix group $\mathcal{C}_{(3)}(\rho) \cong D_{8}$, for which the Molien series and invariant ring were described by Bachoc [1, Theorem 4.4]. 


\section{References}

[1] C. Bachoc, Applications of coding theory to the construction of modular lattices, J. Combin. Theory A 78 (1997), 92-119.

[2] K. Betsumiya and Y. J. Choie, Codes over $\mathbb{F}_{4}$, Jacobi Forms and Hilbert-Siegel modular forms over $\mathbb{Q}(\sqrt{5})$, preprint, 2002.

[3] A. Bonnecaze, A. R. Calderbank and P. Solé, Quaternary quadratic residue codes and unimodular lattices, IEEE Trans. Information Theory 41 (1995), 366-377.

[4] J. Fields, Tables of indecomposable Z/(4) codes, published electronically at http://www.math.uic.edu/ fields/z4/index.html.

[5] P. Gaborit, Mass formulas for self-dual codes over $\mathbb{Z}_{4}$ and $\mathbb{F}_{q}+u \mathbb{F}_{q}$ rings, IEEE Trans. Information Theory 42 (1996), 1222-1228.

[6] P. Gaborit, V. S. Pless, P. Solé and A. O. L. Atkin, Type II codes over $\mathbb{F}_{4}$, Finite Fields Applic. 8 (2002), 171-183.

[7] A. M. Gleason, Weight polynomials of self-dual codes and the MacWilliams identities, in Actes, Congrés International de Mathématiques (Nice, 1970), GauthiersVillars, Paris, 1971, Vol. 3, pp. 211-215.

[8] A. J. Hahn and O. T. O’Meara, The Classical Groups and K-Theory, SpringerVerlag, New York, 1989.

[9] W. C. Huffman and N. J. A. Sloane, Most primitive groups have messy invariants, Advances in Math. 32 (1979), 118-127.

[10] T. Y. Lam, A First Course in Noncommutative Rings, Springer-Verlag, New York, 2nd. ed., 2001.

[11] F. J. MacWilliams and N. J. A. Sloane, The Theory of Error-Correcting Codes, North-Holland, Amsterdam, 1977; 11th impression 2003.

[12] G. Nebe, H.-G. Quebbemann, E. M. Rains and N. J. A. Sloane, Generalized doubly-even codes, (submitted) 2003.

[13] G. Nebe, E. M. Rains and N. J. A. Sloane, The invariants of the Clifford groups, Designs, Codes and Cryptography 24 (2001), 99-121.

[14] G. Nebe, E. M. Rains and N. J. A. Sloane, Self-Dual Codes and Invariant Theory, book in preparation, 2003.

[15] H.-G. Quebbemann, On even codes, Discrete Math. 98 (1991), 29-34. 
[16] E. M. Rains and N. J. A. Sloane, Self-dual codes, in Handbook of Coding Theory, ed. V. Pless and W. C. Huffman, Elsevier, Amsterdam, 1998, pp. 177-294.

[17] G. C. Shephard and J. A. Todd, Finite unitary reflection groups, Canad. J. Math. 6 (1954), 274-304. 